\title{
An exploration of fetish social networks and communities.
}

\author{
Damien Fay, Hamed Haddadi, Michael C. Seto \\ Han Wang, and Christoph Kling \\ Department of Computing, Bournemouth, UK \\ School of Elec. Eng. and Comp. Sci., Queen Mary University, UK \\ The Royal's Institute of Mental Health Research, Ottawa, Canada \\ National University of Ireland, Galway \\ The Leibnitz Institute for the social sciences, Cologne, Germany \\ \{dfay.bournemouth.ac.uk\}
}

\begin{abstract}
Online Social Networks (OSNs) provide a venue for virtual interactions and relationships between individuals. In some communities, OSNs also facilitate arranging offline meetings and relationships. FetLife, the worlds largest anonymous social network for the BDSM, fetish and kink communities, provides a unique example of an OSN that serves as an interaction space, community organizing tool, and sexual market. In this paper, we present a first look at the characteristics of European members of Fetlife, comprising 504,416 individual nodes with 1,912,196 connections. We looked at user characteristics in terms of gender, sexual orientation, and preferred role. We further examined the topological and structural properties of groups, as well as the type of interactions and relations between their members. Our results suggest there are important differences between the FetLife community and conventional OSNs.
\end{abstract}

Keywords: Social network properties, sexuality, topic modelling

\section{Introduction}

Social interaction is motivated at the individual level in need for power, prestige and approval [22] which are expressed in modern life in activities such as business, friendship/emotional learning exchange, and knowledge exchange; and from an evolutionary perspective the need to seek a mate. This latter function of a social network is known as the sexual market and every social network has a secondary function as a sexual market, although disaggregating this function from others can be challenging [12]. In the last decade, Online Social Networks (OSNs) have become a focal point of the web and the most popular activity of individuals online. There are a large number of popular OSNs and a large body of research focuses on a variety of OSNs. Despite a large number of papers on analysis of large scale OSNs [14,2], and a large number of social science papers on social relationships, sexuality and orientations [15][10], there have not been 
any academic papers which have examined online social networks focused on variations in sexual orientations and interests.

In this paper, we take a first look at the anonymised profiles of the European users of the most popular fetish website, and ask if the characteristics of the network are different from those of a conventional OSN. This is a rich dataset of over half a million users and captures patterns of traditionally secret interests and behaviours. We do so by comparing the topological characteristics with those of popular social networks as reported by Mislove et al. [14]. We choose this online fetish network as it is oriented towards friendships, social groups, and arranging events, where the social is primary and sexual market is secondary but explicitly included (unlike, say, Facebook or other non-dating OSNs). It is important to social scientists and psychologists to understand whether a social network is also present or not required. As FetLife reveals sexuality in a social context it allows us to understand sexual networks in a way that dating sites such as Tinder, Grindr etc might not allow; this is also vital for creating models for the spread of sexually transmitted infections [17].

We use our large dataset to assess the properties of these multi-relationship networks, where a user can have a number of different types of relationships with others. ${ }^{1}$ We base our analysis of the structure of the graph on work by Laumann et al. [12], who use self reports and assess individuals' roles and economic factors in sex markets, using four neighbourhoods in Chicago and highlight the role of brokers and third parties in this exchange. Our dataset uses the largest broker out there, the world's most popular fetish site, as a benchmark for analysis of the online version of this market. Understanding the nature of the interactions is also important for real and cyber crime investigations, as the privacy and safety of users could also be compromised by malicious users of such websites. $^{2}$

\section{Online Fetish Networks}

We collected our data from FetLife, ${ }^{3}$ the most popular Social Network for the BDSM, Fetish, and kink communities, with millions of users worldwide. The fetish community has grown rapidly in recent years and now consists of a diverse collection of people whose interests cover a broad spectrum including, fashion, burlesque, a nightclub scene, particular types of music and of course a focus on sexual experimentation. As in Facebook, the interaction of the community is both real-world and virtual with a large collection of real-world events attended by members; contrary to expectations, FetLife it is not a paid dating website. For example, there is no "search" functionality within the website for specific types of members, e.g., based on interests, or over user information fields (height, weight, age, location, fetish commonalities, other personal information). However

\footnotetext{
${ }^{1}$ In the interest of space and scientific focus, we encourage the readers to see $[13,16]$ for a description of different types of fetish relationships.

2 http://sexandthe405.com/fetlife-is-not-safe-for-users/

${ }^{3}$ https://fetlife.com/
} 
the site is used as a bootstrapping mechanism for social events, workshops, and parties which are organised regionally. Members create a personal profile, similar to most OSNs, specify their gender, age, role, orientation, and list the fetishes they are interested in or are curious about. The users are organised into tens of thousands of groups, and thousands of events are arranged annually through the website. Users pay particular attention to the experience of the group members and event organisers and hence these individuals play a central role in the community. In essence, FetLife is a niche OSN. BDSM is a sexual interest or subculture attractive to a minority [18]. What makes FetLife unique particularly interesting for OSN analysts is that this website observes sexual interaction (present in dating websites, absent in typical social networks such as Facebook) but in the presence of a social context (absent in dating websites).

\section{Data collection}

We collected our data from the European members of FetLife during the early months of 2014. The data includes anonymized (at the time of collection) user IDs, relationship types, and number of friends. In order to comply with the website policy and ethics approval requirements, we did not crawl any names, details of friends, pictures, posts, or other personally identifiable information available on the site. Since it is mandatory for users to be a member of a single geographic area (usually county/borough level depending on the population density), our crawler used the location area codes of the website as its seed and we collected the mentioned details about every single individual in the European section of the website.

Overall, there are 504,416 individual nodes in our dataset, with 1,912,196 connections. The main connected component is comprised of just over $156 \mathrm{~K}$ nodes, and the rest of the users are mainly isolated or small groups of maximum size 20. At the time of collection, there were 35,153 groups in the dataset, with just over $26 \mathrm{k}$ single nodes. Although this is a sample of the population and only captures the individuals who chose to be on a fetish OSN, this data is more inclusive and less biased than the offline club members or those who self-identify for sample surveys in existing literature $[4,18]$. The perceived anonymity online and low (essentially zero) cost of entry into Fetlife means more individuals might be active online than joining actual clubs, going to local BDSM themed parties or self-identifying to researchers at universities.

\section{Demographic analysis}

In this section we document the demographics of the fetish network such as gender, sexual preference, and connections. The identity acronyms are defined as follows: $\mathrm{M}=$ cis male; $\mathrm{F}=$ cis female; $\mathrm{TV}=$ transvestite; $\mathrm{TS}=$ transsexual, which can be further distinguished into male-to-female transsexuals (MtF or trans females) and female-to-male transsexuals (FtM or trans males); Kajira/Kajiru are slave girl/boy; $\mathrm{I}=$ intersex, $\mathrm{B}=$ butch, Fem $=$ Femme. If not 


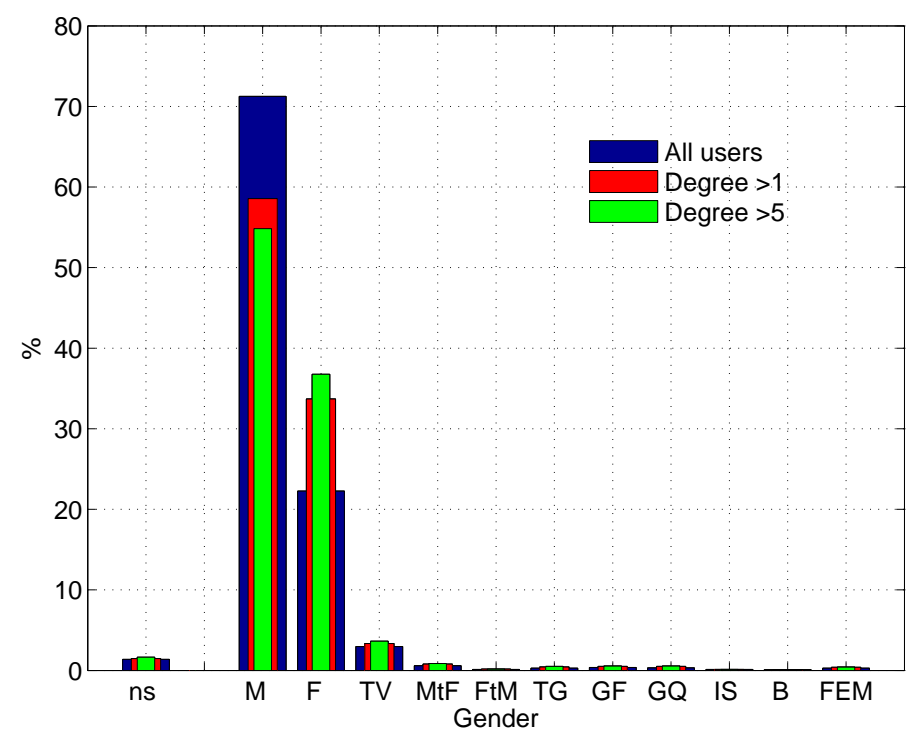

Fig. 1: Distribution of genders for all users, users with $>1$ friends, and $>5$ friends.

otherwise stated, Trans $=$ trans females and TVs. GF $=$ gender fluid and GQ $=$ gender queer, referring to persons who do not identify as male or female or see themselves as having aspects of both genders. We first look at the gender demographics of the users as a whole. As mentioned previously, there are larger number of users with no friends than would otherwise be expected. Figure 1 shows the distribution of user gender for all users. When the singletons have been removed, the gender distribution changes drastically; most of those with few or no friends are male (Figure 3 shows that in addition they tend to be heterosexual males). When we have taken out those with fewer than 5 friends then the gender distribution is quite even with (non-cis) $54 \%$ male, $40.5 \%$ female and other (cis) genders making up the remainder. Figure 2 diagrammatically is a graph indicative of the potential partners of different genders taking orientations into account. The graph is quite complicated with heterosexual relationships being reciprocal, gay relationships being homophilic (manifesting as graph loops), several uni-directional links (ex: a lesbian may consider a straight girl as a potential partner but this may not be reciprocated). In essence the sexual market which presents itself is neither bipartite nor undirected and so defies OSN analysis such as that in [11].

In Table 1 we examine the congruence of users with respect to gender and orientation. The network congruence is defined in [20] as the probability of one's friends having the same attributes or related attributes. That is, we wish to ask if people of a particular gender and orientation have preference for another gender. The results here show a strong preference in accordance with the graph 


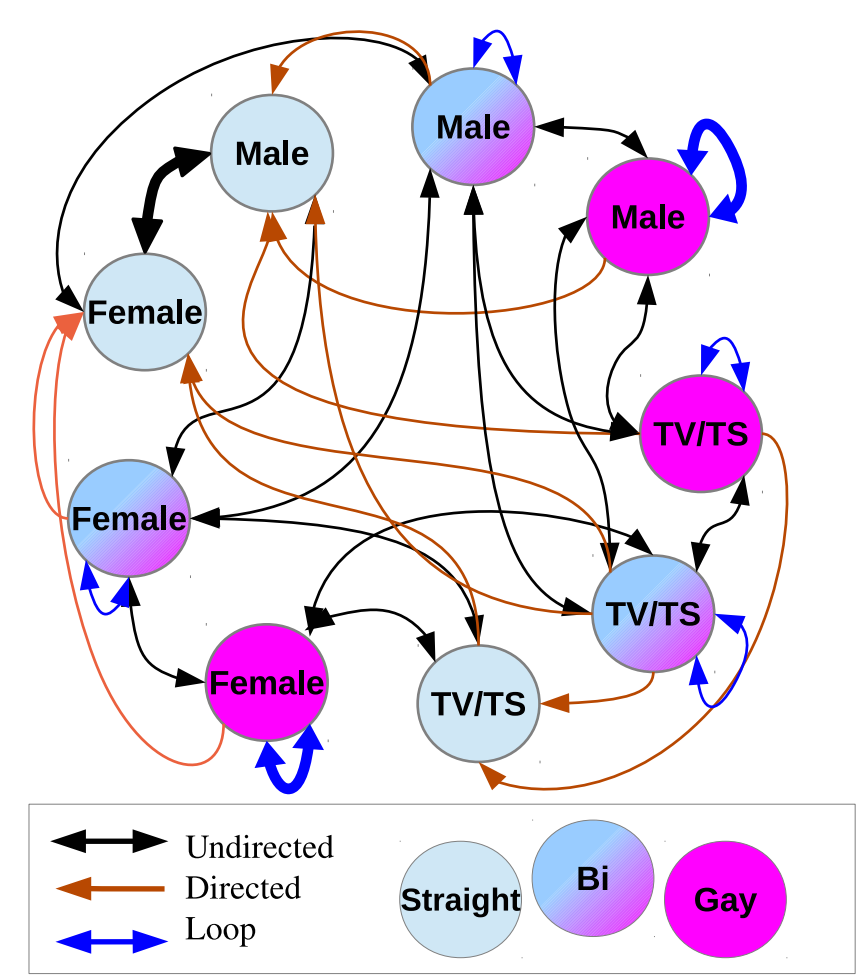

Fig. 2: Graph of potential partners. Note that some links are directed, and the graph is not complete.

shown in Figure 2. For example, gay men have on average $32 \%$ of their friends composed of gay men, far exceeding the population average of $1.5 \%$. A straight female will have $57 \%$ of her friends as straight males, higher than the population average (39\%), slightly higher than the bisexual female average (53.4\%) and significantly higher than the gay female average $(42 \%)$. Overall the platonic relationships (in blue) are mostly lower than the population averages (exceptions are gay females who have a slightly higher than population average friendship with straight males; and gay trans to gay females). For straight females $73 \%$ of their friends are straight/bisexual males. For straight males, $61.2 \%$ of their friends are (straight/bisexual) females. This would strongly suggest a sexual market (for hetero- and bi-sexual people) as it implies not only a bias towards the opposite sex but also competition (see [1] for an excellent discussion). It implies that a male is less likely to be friends with the male friends of his female friends than he would with a person from the population as a whole. That is, there would appear to be evidence of competition between males (and vice versa; also between females). This behaviour online complements existing research in 
the literature that shows atypical sexual interests are more common in men than in women [4].

\begin{tabular}{|c|c|c|c|c|c|c|c|c|c|}
\hline & M-S & M-Bi & M-G & F-S & F-Bi & F-G & Tr-S & Tr-Bi & Tr-G \\
M-S & 27.1 & 7.1 & 0.7 & $\mathbf{1 7 . 1}$ & 44.1 & 1.5 & 0.3 & 1.9 & 0.3 \\
M-Bi & 26.8 & 12.9 & 3.0 & 12.7 & 36.4 & 1.4 & 0.6 & 5.6 & 0.6 \\
M-G & 23.8 & 27.6 & $\mathbf{3 2 . 9}$ & 2.7 & 8.4 & 0.8 & 0.2 & 3.1 & 0.5 \\
F-S & $\mathbf{5 7 . 0}$ & 16.3 & 0.4 & 6.5 & 14.9 & 0.9 & 0.6 & 3.2 & 0.3 \\
F-Bi & 53.4 & 16.8 & 0.4 & 5.4 & 18.7 & 1.4 & 0.5 & 3.1 & 0.3 \\
F-G & 42.9 & 12.9 & 0.8 & 6.2 & 26.8 & $\mathbf{6 . 1}$ & 0.5 & 3.0 & 0.8 \\
Tr-S & 25.6 & 12.2 & 0.6 & 9.4 & 22.8 & 1.1 & 3.5 & 22.9 & 1.8 \\
Tr-Bi & 23.9 & 15.7 & 1.0 & 7.0 & 18.7 & 0.9 & 3.0 & 27.6 & 2.3 \\
Tr-G & 29.9 & 16.1 & 1.5 & 5.8 & 17.8 & 2.3 & 2.2 & 21.4 & $\mathbf{3 . 0}$ \\
\hline All & 39.1 & 13.2 & 1.5 & 10.3 & 28.8 & 1.4 & 0.6 & 4.7 & 0.5 \\
\hline
\end{tabular}

Table 1: Congruency of gender and orientation: $\{$ Male,Female,Trans $\} \times$ $\{$ Straight,Bisexual,Gay\}. Potential partners in black, platonic in blue, and conventional partners in bold.

We compared our results with that of Pokec, a large European OSN of over 1.6 million subscribers with gender specifications [21]. In Pokec, male members are $49 \%$ and $51 \%$ likely to connect to males and females respectively, while these figures are $55 \%$ and $45 \%$ for females connecting to females and males respectively. This is a rather balanced ratio and in a rather significant contrast with the fetish network's data which has a strong bias towards the opposite sex, further supporting the sexual market social network hypotheses. It is worth noting that, although men are more active users of cybersex channels, significantly more women than men state that their online sexual activities had led to real-life sexual encounters [19].

For the TV, MtF, FtM, and TG users there appears to be a strong preference towards friends of the same gender. For example, a TV will tend to have $29.5 \%$ friends, far above the population average of $4.7 \%$. However, it is interesting to note that while there is a strong bias towards people of the same gender the majority of friends still come from other genders; there is no evidence to support the idea of closed minority gender communities.

Figure 3 shows the distribution of sexual orientations of users. Of the users, $45 \%$ describe themselves as heterosexual while less than $5 \%$ describe themselves as exclusively gay or lesbian. Large survey-based studies show that BDSM activities are more common among non-heterosexual individuals (gay, lesbian or bisexual) [18].

Figure 4 shows the distribution of user roles. Some of these roles are quite similar and interestingly the dominant roles (Dom, Domme, Mistress, Master) 


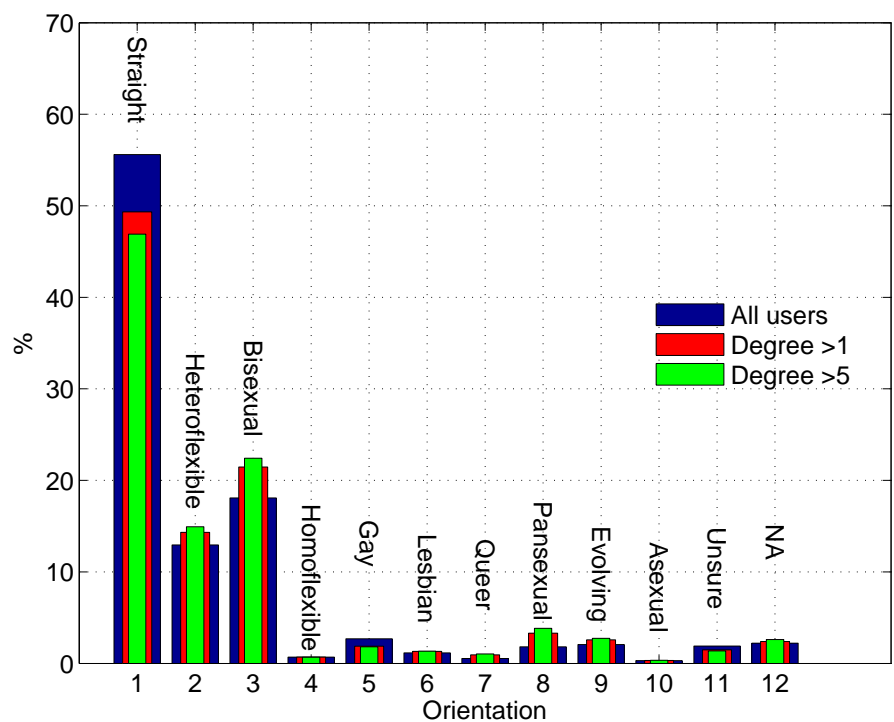

Fig. 3: Distribution of orientations for all users, users with $>1$ friends, and $>5$ friends.

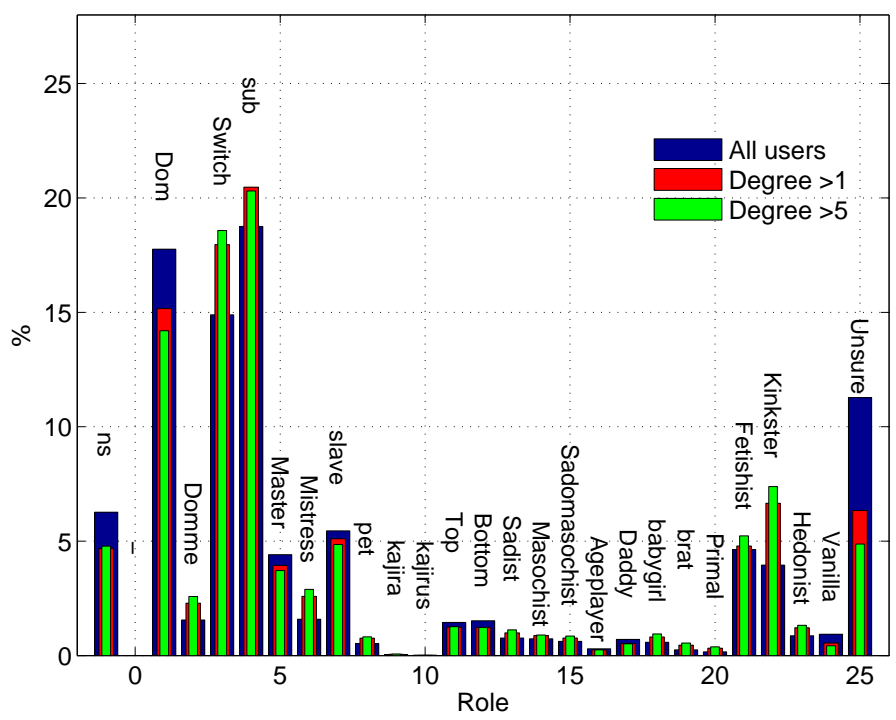

Fig. 4: Distribution of roles for all users, users with $>1$ friends, and $>5$ friends.

take up $23.39 \%$ of the roles while the submissive roles (Sub, Slave) take up $25.15 \%$ of the users, a remarkable balance. 
For comparison we examined the fetish network structure with those of standard OSNs (YouTube, Flickr, LiveJournal and Orkut) following the analysis, and using results, of [14]. We then look into more complex measures such as the average path length, Joint Degree Distribution (JDD, a measure of connectivity of one's neighbours), clustering coefficient (measure of density of triangular ties between adjacent nodes), and assortativity, which indicate the relations between the nodes on a local basis. We also explore the hierarchical structure of the network using k-cores and Kernel density estimation. ${ }^{4}$ The degree distribution is shown in Figure 5, and is unremarkable except that there is a larger than expected number of users with low degree. These are removed when we examine the main component of the graph (as previously mentioned these users would appear to be lurkers; mostly heterosexual males who do not participate in the social network). Figure 6 shows the distribution of the degrees of friends of degree $k$. Again this is unremarkable and similar may be found in [14]. Finally, Table 2 gives a summary of common network measures. ${ }^{5}$ The main conclusion is that FetLife has a very similar structure to most OSN's.

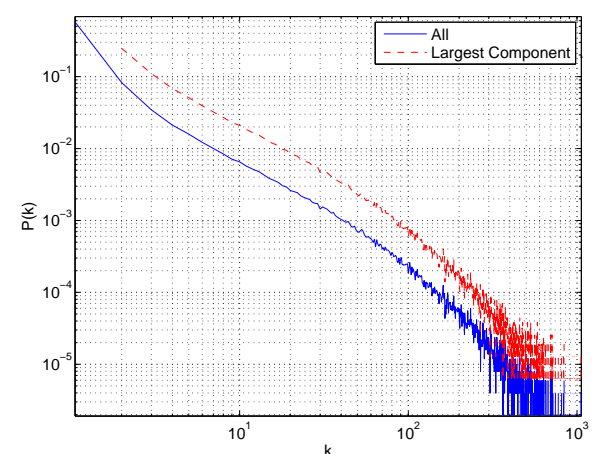

Fig. 5: Degree distribution of the network and LCC.

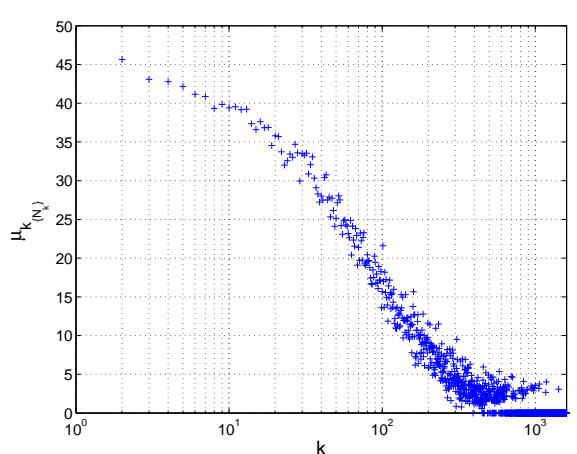

Fig. 6: Semi-log plot of the average degree of the friends of users with degree of $k$.

Figure 7 shows the k-core of removal rate and that the network is highly resilient to removal of high degree nodes. In fact we could remove the top $10 \%$ of the nodes and only lose $30 \%$ off the largest connected component. This indicates that the network consists of lots of small connections between people ignoring the core. The large number of small groups and local clusters, as opposed to large inter-mixed nodes, is the main reason behind this effect, which has also recently been observed in the Internet topology [8]. In FetLife, the events and connections

\footnotetext{
${ }^{4}$ A complete explanation of the theoretical definitions and implications of these measures is available in [7] and [9].

${ }^{5}$ We assume that the reader is familiar with standard network measures (a good overview may be found in $[6,7,9])$
} 


\begin{tabular}{|l|c|c|c|c|c|c|}
\hline & $\alpha$ & avg path length & Radius-Diameter & assortativity & scale free metric & avg clustering coefficient \\
Fetish & 2.98 & 4.05 & $7-11$ & -0.01 & 0.0031 & 0.15 \\
Flickr & 1.74 & 5.67 & $13-27$ & 0.202 & 0.49 & 0.313 \\
Livejournal & 1.59 & 5.88 & $12-20$ & 0.179 & 0.34 & 0.330 \\
Orkut & 1.50 & 4.25 & $6-9$ & 0.072 & 0.36 & 0.171 \\
Youtube & 1.63 & 5.10 & $13-21$ & -0.033 & 0.19 & 0.136 \\
Web & 2.57 & 16.12 & $475-905$ & -0.067 & - & 0.081 \\
\hline
\end{tabular}

Table 2: Network Measures from the fetish and ordinary OSNs.

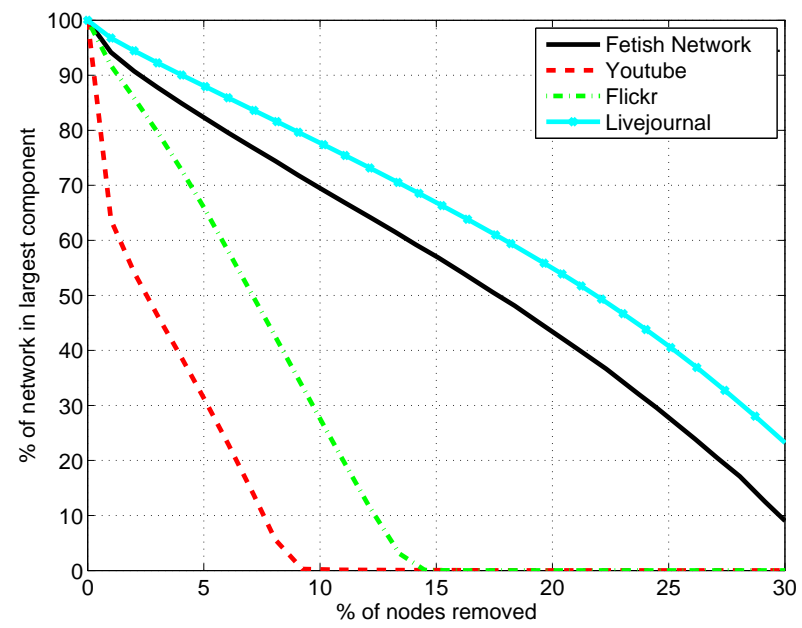

Fig. 7: Percentage of main component remaining after removal of the highest degree users.

are centred around local events, meetings, and workshops. Although a direct search function is not available, many users of the website use the network as a portal to bootstrap their fetish sex life. Hence the global connectivity is not as important as traditional OSNs such as Twitter and Facebook, and far from content-centric OSNs such as Flickr and YouTube.

\section{Community Detection}

From the analysis above (Table 1 in particular) we see a network where there are connections for many reasons. Some connections are created for sexual attraction, others are purely social. Within the sexual attractions there is homophilic and heterophilic factors and in addition there are heterophilic sexual connections to do with a persons role (a dominant person would in particular like a submissive person). Constructing a community detection algorithm for this set is thus a complicated task requiring not just knowledge of the links in the network but also the attributes associated with those links. A recent paper by Yang et. al. [23] proposed the CESNA model (Community Detection in Networks with Node Attributes). This model is generative and based on the assumption that 
a link/edge is created between two users/vertices if they share membership of a particular community. In addition, vertices may be members of several independent communities such that the probability of creating an edge is 1 minus the probability that no edge is created in any of their common communities as:

$$
P_{u \rightarrow v}=1-\sum_{c \in C} 1-e^{-F_{u c} F_{v c}}
$$

where $F_{u c}$ is the potential of vertex $u$ to community $c, P_{u \rightarrow v}$ is the probability that $u$ connects to $v$, and $C$ is the set of all communities. In addition, it assumed that the attributes of a vertex are also generated from the communities they are members of and so the graph and the attributes are generated jointly by some underlying unknown community structure. Specifically the attributes are assumed to be binary (present or not present) and are generated according to a Bernoulli process according to:

$$
X_{u k} \sim \mathcal{B}\left(Q_{k}\right)
$$

where $Q_{k}=\frac{1}{1+e^{-\sum_{c \in C} W_{k c} F u c}}, W_{k c}$ is a weight matrix $\in \mathbb{R}^{N \times|C|}$, which defines the strength of connection between the $N$ attributes and the $|C|$ communities. $W_{k c}$ is central to the model and is a set of logistic model parameters and together with the number of communities, $|C|$, forms the set of unknown parameters for the model. Parameter estimation is achieved by maximising the likelihood of the observed graph (i.e. the observed connections) and the observed attribute values given the membership potentials and weight matrix. As the edges and attributes are created from conditionally independent (given $W$ ) draws the log likelihood may be expressed as a summation of three different events:

$$
\begin{array}{r}
\log P(G, X \mid F, W)=\sum_{u, v \in E} \log \left(1-e^{-F_{u} F_{v}^{T}}\right)-\sum_{u, v \notin E} F_{u} F_{v}^{T} \\
+\sum_{u, k} X_{u k} \log Q_{u k}+\left(1-X_{u k}\right) \log \left(1-Q_{u k}\right)
\end{array}
$$

where the first term on the right hand side is the probability of observing the edges in the network, the second term is the probability of observing the nonexistent edges in the network, and the third term are the probabilities of observing the attributes; under the model. The parameters of the model may be estimated by a two stage procedure with full details provided in [23]. The data used in the community detection for this network consists of the main component of the network together with the attributes \{Male, Female, Trans, GQ\} together with orientations \{ Straight, Bisexual, Gay $\}$ and roles $\{$ submissive, dominant, switch $\}$ for a total of 10 binary attributes. The optimum number of communities was found using CESNA to be 10. Table 3 shows the values of the weights vector, $W_{k c}$. This table shows a mixed story. The first community has a shared affinity to each gender, and then represents dominant bisexuals. 
The second community is mainly male and dominant. In fact the dominant role tends to be the strongest in community detection. A notable exception is with community 6 which seems to be mostly composed of those with gay orientation. Overall the results are quite mute and would suggest that the CESNA model is incapable of extracting the communities based on the known structure shown in Figure 2. The reason for this is that Equation 5 assumes that those in the same community have an affiliation for each other. In the sexual market this is not necessarily the case (though it may explain the strong coefficient in community 6 for gay people). There is no underlying method in the model to express (for example) that a woman in the straight female community is more likely to connect to a member of the straight male community than she is to her own community. Another example is that dominant individuals will tend to connect with submissive individuals. Indeed there is no way of creating pseudo-attributes that would allow separation of these heterophilic communities.

\begin{tabular}{|c||c|c|c|c|c|c|c|c|c|c|}
\hline Attribute & M & F & Tr & GQ & Str8 & Bi & Gay & Dom & Sub & Switch \\
\hline C1 & -0.6 & 0.6 & -0.6 & -0.6 & 0.1 & $\mathbf{1 . 5}$ & -0.8 & $\mathbf{1 . 9}$ & -0.2 & 0.5 \\
C2 & $\mathbf{- 0 . 7}$ & 0.4 & -0.2 & 0.2 & 1.1 & -0.4 & -0.0 & $\mathbf{- 1 . 2}$ & $\mathbf{- 1 . 0}$ & -0.5 \\
C3 & -0.8 & 0.8 & -0.8 & 0.3 & -0.1 & -0.4 & 0.0 & $\mathbf{3 . 6}$ & 0.0 & $\mathbf{1 . 0}$ \\
C4 & -0.4 & 0.6 & -0.5 & -0.4 & 0.5 & $\mathbf{1 . 0}$ & -0.3 & -0.5 & -0.5 & 0.6 \\
C5 & -0.6 & 0.4 & -0.3 & -0.3 & $\mathbf{0 . 9}$ & 0.3 & 0.2 & $\mathbf{- 1 . 2}$ & -0.8 & 0.3 \\
C6 & -0.3 & 0.2 & -0.2 & 0.3 & 0.3 & 0.0 & $\mathbf{1 . 5}$ & -0.5 & -0.0 & 0.4 \\
C7 & -0.6 & 0.3 & -0.2 & 0.4 & 0.5 & 0.0 & -0.7 & -0.8 & -0.6 & $\mathbf{1 . 1}$ \\
C8 & -0.3 & 0.2 & -0.6 & 0.5 & 0.3 & -0.3 & -0.6 & -0.5 & $\mathbf{2 . 2}$ & 0.9 \\
C9 & 0.1 & -0.1 & -0.1 & -0.1 & -0.1 & 0.2 & -0.1 & -0.1 & 0.6 & -0.0 \\
C10 & 0.0 & 0.0 & -0.0 & 0.0 & -0.0 & -0.0 & -0.0 & -0.0 & 0.0 & -0.0 \\
\hline
\end{tabular}

Table 3: Weight matrix for 10 communities detected using 10 attributes.

\section{Conclusion}

The importance of the diversity of online sexual contacts is of growing importance [3] and several studies have looked at how it effecting our sexuality particularly in the West [5]. The fetish network examined here has many of the functionalities of mainstream OSNs: there are friendship links, relationships, interests, groups and events. The network is a valuable source of information as it is neither a dating website nor a standard OSN. Rather, it is an OSN where the sexual market aspects of the network have been amplified. The picture that emerges is one of complex hetero and homo-philic interacting communities and in addition, people that form friendships which are purely platonic. However, we also observe that while these factors are clearly evident in Table 1 that the CESNA community detection algorithm is unable to clearly distinguish the groups. This is not a failing of the algorithm, this algorithm advances part of 
the way by jointly modelling edge probabilities and node attributes (as do similar more recent algorithms [24]) but rather an indication that more complex community interactions are required to properly represent the rainbow of sexual preferences, orientations and genders present in our society.

\section{References}

1. D. M. Buss. The evolution of desire: Strategies of human mating. New York: Basic Books., 2003.

2. M. Cha, F. Benevenuto, H. Haddadi, and K. Gummadi. The world of connections and information flow in twitter. Systems, Man and Cybernetics, Part A: Systems and Humans, IEEE Transactions on, 42(4):991-998, 2012.

3. A. Cooper, J. Morahan-Martin, R. M. Mathy, and M. Maheu. Toward an increased understanding of user demographics in online sexual activities. Journal of Sex 8 Marital Therapy, 28(2):105-129, 2002. PMID: 11894795.

4. S. J. Dawson, B. A. Bannerman, and M. L. Lalumière. Paraphilic interests: An examination of sex differences in a nonclinical sample. Sexual abuse: a journal of research and treatment, 2014.

5. N. M. Dring. The internets impact on sexuality: A critical review of 15 years of research. Computers in Human Behavior, 25(5):1089 - 1101, 2009. Including the Special Issue: Design Patterns for Augmenting E-Learning Experiences.

6. D. Easley and J. Kleinberg. Networks, Crowds, and Markets: Reasoning About a Highly Connected World. Cambridge University Press, 2010.

7. D. Fay, H. Haddadi, A. Thomason, A. W. Moore, R. Mortier, A. Jamakovic, S. Uhlig, and M. Rio. Weighted spectral distribution for internet topology analysis: theory and applications. Networking, IEEE/ACM Transactions on, 18(1):164-176, 2010.

8. H. Haddadi, D. Fay, S. Uhlig, A. Moore, R. Mortier, and A. Jamakovic. Mixing biases: Structural changes in the as topology evolution. In Traffic Monitoring and Analysis, pages 32-45. Springer Berlin Heidelberg, 2010.

9. H. Haddadi, D. Fay, S. Uhlig, A. Moore, R. Mortier, A. Jamakovic, and M. Rio. Tuning topology generators using spectral distributions. In Performance Evaluation: Metrics, Models and Benchmarks, pages 154-173. Springer Berlin Heidelberg, 2008.

10. C. C. Joyal, A. Cossette, and V. Lapierre. What exactly is an unusual sexual fantasy? The Journal of Sexual Medicine, 12(2):328-340, 2015.

11. J. Kunegis, G. Grner, and T. Gottron. Online dating recommender systems: The split-complex number approach. In Proc. Workshop on Recommender Systems and the Social Web, pages 37-44, 2012.

12. E. Laumann. The Sexual Organization of the City. Studies in Communication, Media, and Public Opinion. University of Chicago Press, 2004.

13. A. Lewis. Just your average joe: getting to know a lifestyle kinkster. Counselling Australia, 11(2), 2012.

14. A. Mislove, M. Marcon, K. P. Gummadi, P. Druschel, and B. Bhattacharjee. Measurement and analysis of online social networks. In Proceedings of the 7th ACM SIGCOMM Conference on Internet Measurement, IMC '07, pages 29-42, New York, NY, USA, 2007. ACM.

15. G. Mollenhorst, B. Vlker, and H. Flap. Social contexts and personal relationships: The effect of meeting opportunities on similarity for relationships of different strength. Social Networks, 30(1), 2008. 
16. T. Mrozewski. How do kinky people know what they know? The information behaviour of BDSM practitioners. 2013.

17. A.-M. Niekamp, L. A. Mercken, C. J. Hoebe, and N. H. Dukers-Muijrers. A sexual affiliation network of swingers, heterosexuals practicing risk behaviours that potentiate the spread of sexually transmitted infections: A two-mode approach. Social Networks, 35(2):223 - 236, 2013. Special Issue on Advances in Two-mode Social Networks.

18. Richters, Juliet, D. Visser, O. Richard, Rissel, E. Chris, Grulich, E. Andrew, Smith, and M. A. Anthony. Demographic and Psychosocial Features of Participants in Bondage and Discipline, Sadomasochism or Dominance and Submission (BDSM): Data from a National Survey. The Journal of Sexual Medicine, 5(7), jul 2008.

19. J. P. Schneider. A qualitative study of cybersex participants: Gender differences, recovery issues, and implications for therapists. Sexual Addiction $\mathcal{E}$ Compulsivity: The Journal of Treatment \& Prevention, 7(4):249-278, 2000.

20. A. Singla and I. Weber. Camera brand congruence in the flickr social graph. In Proceedings of the Second ACM International Conference on Web Search and Data Mining, WSDM '09, pages 252-261, New York, NY, USA, 2009. ACM.

21. L. Takac and M. Zabovsky. Data analysis in public social networks. In International Scientific Conference $\mathcal{E}$ International Workshop Present Day Trends of Innovations, May 2012.

22. J. Turner. A Theory of Social Interaction. Polity Press, 1988.

23. J. Yang, J. J. McAuley, and J. Leskovec. Community detection in networks with node attributes. In International Conference on Data Mining, 2013.

24. F. Zhang, J. Li, F. Li, M. Xu, R. Xu, and X. He. Community detection based on links and node features in social networks. In X. He, S. Luo, D. Tao, C. Xu, J. Yang, and M. Hasan, editors, MultiMedia Modeling, volume 8935 of Lecture Notes in Computer Science, pages 418-429. Springer International Publishing, 2015. 